\title{
Glucagon-like Peptide-1 Receptor Agonist Treatment Attributes Important to Injection-Naive Patients with Type 2 Diabetes Mellitus: A Multinational Preference Study
}

Lei Qin · Stephanie Chen · Emuella Flood · Alka Shaunik ·

Beverly Romero · Marie de la Cruz · Cynthia Alvarez · Susan Grandy

Received: December 7, 2016/Published online: February 2, 2017

(C) The Author(s) 2017. This article is published with open access at Springerlink.com

\begin{abstract}
Introduction: Glucagon-like peptide-1 receptor agonists (GLP-1RAs) differ in efficacy, side effects, dosing frequency, and device-related attributes. This study assessed the relative importance of treatment-related attributes in influencing preferences for GLP-1RAs among injection-naive patients with type 2 diabetes mellitus (T2DM).

Methods: Injection-naïve T2DM patients from five countries completed a Web-based discrete choice experiment (DCE) survey. Patients chose between hypothetical treatment profiles reflecting important and differentiating attributes of GLP-1RAs. Eight attributes were
\end{abstract}

Enhanced content To view enhanced content for this article go to http://www.medengine.com/Redeem/ 8687F0604C4A11A3.

Electronic supplementary material The online version of this article (doi:10.1007/s13300-017-0230-2) contains supplementary material, which is available to authorized users.

L. Qin $(\bowtie) \cdot$ S. Chen · A. Shaunik · S. Grandy AstraZeneca, Gaithersburg, MD, USA

e-mail: lei.qin@astrazeneca.com

E. Flood - B. Romero · M. de la Cruz

ICON plc, Clinical Outcomes Assessments,

Gaithersburg, MD, USA

C. Alvarez

ICON plc, Medical Affairs Statistical Analysis,

San Diego, CA, USA included: efficacy, side effects, device size, needle size, titration, preparation, evidence of long-term efficacy/safety, and dosing frequency. Odds ratios (ORs) and 95\% confidence intervals were calculated using a conditional logit model to indicate the likelihood of choosing a treatment with a given attribute level versus a reference attribute level. The influence of individual attributes when considering full treatment profiles was examined using exenatide once weekly (QW) and liraglutide once daily (QD) as case examples.

Results: A total of 1482 patients with T2DM completed the DCE survey. Side effects, efficacy, and dosing frequency were the three most important attributes influencing preferences; needle size, device size, and required preparation were least important. Total sample analysis indicated that a profile of GLP-1RA approximating exenatide QW (single pen) was preferred over a profile approximating liraglutide QD (OR 3.36; $p<0.001)$, when efficacy was assumed to be equal.

Conclusion: The most influential drivers of treatment preferences for a hypothetical GLP-RA profile were side effects, efficacy, and dosing frequency among injection-naïve T2DM patients. Preference elicitation can promote patient-centered care and inform new generations of T2DM treatments, which can lead to improved adherence and health outcomes. 
Keywords: Conjoint; Device; Dosing frequency; Exenatide; Liraglutide; Type 2 diabetes mellitus

\section{INTRODUCTION}

Type 2 diabetes mellitus (T2DM) is a progressive metabolic disorder characterized by hyperglycemia resulting from a progressive loss of insulin secretion on the background of insulin resistance $[1,2]$. The International Diabetes Federation has estimated that in 2015 the prevalence of diabetes was $8.8 \%$ and that this will have increased to $10.4 \%$ by 2040 [3]. Approximately $87-91 \%$ of all people with diabetes in high income countries are estimated to have T2DM [3]. Increases in obesity, physical inactivity, urbanization, and an aging and expanding population have contributed to the increasing prevalence of this disease [3-5]. If inadequately managed, diabetes can lead to heart attack, stroke, kidney disease, blindness, and amputation [2, 6, 7].

The majority of T2DM patients require medication for glycemic control, often initiating with oral anti-diabetes drug (OAD) monotherapy and progressing to combination therapy or injectable treatment [7-9]. Medication adherence plays an essential role in overall glycemic control, and helps to reduce the risk of complications, to prevent premature mortality, and to lower overall healthcare costs [10-12]. Nevertheless, adherence rates as low as $45 \%$ have been reported among those with T2DM [13]. Non-adherence has been associated with various treatment-related factors, including complex or frequent dosing, and treatment side effects $[10,13]$. The American Diabetes Association (ADA) and the European Association for the Study of Diabetes (EASD) have emphasized the importance of considering patients' preference for such treatment-related factors when clinical decisions around the management of T2DM are being made [7]. Decisions that are patient centered are likely to improve adherence and consequently clinical outcomes $[10,14]$.

Glucagon-like peptide-1 receptor agonists (GLP-1RAs) are a newer class of injectable T2DM treatments developed to target the incretin system $[15,16]$. Agents in this class vary particularly with respect to key attributes (including dosing regimen and the risk of side effects) which have been associated with patient non-adherence [17-19].

The objective of this study was to examine the relative importance of treatment-related attributes in influencing preferences for GLP-1RAs among injection-naïve patients with T2DM in a wide geographic distribution [the UK, Germany, China, Brazil, and Japan]. Two commonly prescribed GLP-1RAs that were available at the time of the study and that differ in terms of key attributes influencing adherence (regimen and risk of side effects) were selected to explore preferences for treatments that differed with respect to these key features: once-weekly (QW) exenatide, the first approved once-weekly GLP-1RA treatment, and oncedaily (QD) liraglutide, the most commonly prescribed once-daily GLP-1RA treatment.

\section{METHODS}

\section{Study Design}

This was a cross-sectional, descriptive study involving a one-time, Web-based discrete choice experiment (DCE) survey completed by injection-naïve T2DM patients.

DCE, a type of conjoint analysis, is a robust methodology to elicit consumer preferences and is increasingly being used to examine patient preferences for healthcare interventions $[20,21]$. DCE assumes that any product or service can be described by its characteristics (or attributes) and that an individual values the product or service on the basis of the levels of these attributes. In a DCE, individuals are presented with hypothetical descriptions of products and are asked to choose their preferred product on the basis of the levels of attributes presented. Response data are modelled within a benefit (or satisfaction) function, which provides information on whether or not the given attributes are important, the relative importance of attributes, the rate at which individuals are willing to trade between attributes, and 
overall benefit scores for alternative products [20].

In this study, the DCE survey was completed by adult T2DM patients in the UK, Germany, China, Brazil, and Japan. Eligibility was determined by the following criteria: patient-reported diagnosis of T2DM; age 18 years or older; adequate written and oral fluency in the target language; willing and able to complete a 30-min online survey to answer questions about treatment experiences and preferences; have access to the Internet; willing to provide informed consent; currently on an OAD; and no experience with injection treatment for T2DM (i.e., injection-naïve). Eligible participants were identified by a market research recruitment agency using multiple methods including searching databases of patients who had agreed to be contacted for participation in such research studies, referrals from clinicians and patient associations, and advertising in targeted publications. This study aimed to recruit a diverse sample with respect to age, gender, and education status.

The survey was programmed and hosted by Global Perspectives (Berkshire, UK) on a secure server. Interested participants were provided with an Internet link to the online Web-based DCE survey. All participants were screened online to ensure that they met the specific eligibility criteria. All eligible patients provided online consent through the Web link prior to completing the survey. Those who completed the survey received compensation for their participation.

The study protocol was approved by the Salus International Review Board (Austin, TX, USA). All procedures followed were in accordance with the ethical standards of the responsible committee on human experimentation (institutional and national) and with the Helsinki Declaration of 1964, as revised in 2013.

\section{Survey Development}

A literature review and qualitative research with clinicians and T2DM patients were conducted to inform attribute selection and survey development. Open-ended, one-on-one interviews were conducted with 50 T2DM patients (from the five target countries) with experience taking GLP-1RAs or who were injection-naïve to identify and examine treatment attributes important to T2DM patients [22].

On the basis of the findings from the literature and qualitative research, eight attributes related to treatment efficacy, safety, tolerability, and convenience were included in the DCE survey: efficacy [improvement in glycated hemoglobin (HbA1c)], common GLP-1RA side effects (nausea, diarrhea, vomiting, and injection site nodules), injection device size, needle size, need for titration, required injection preparation (associated with vial/syringe, single-use pen, multi-use pen, or auto-injector), evidence of long-term efficacy/safety, and dosing frequency (daily or weekly). The US Food and Drug Administration-approved labels of two common GLP-1 RAs [23, 24] were reviewed to provide profiles reflecting two hypothetical treatments (Fig. 1). One treatment was a profile of a daily injectable, approximating liraglutide QD, the most commonly prescribed daily GLP-1RA treatment. The other treatment was an injectable approximating exenatide $\mathrm{QW}$, the first QW GLP-1RA treatment. Exenatide QW is delivered once weekly, does not require titration, and uses a thicker, longer needle. Delivery of exenatide was specified as either using a single-use pen device or via an auto-injector. The single-use pen device contains exenatide microspheres and lipid-based diluent in separate chambers, requiring careful preparation of the medication, while the auto-injector has the exenatide microspheres and lipid-based diluent prefilled in one chamber. Exenatide QW can also be delivered in a single-dose tray (vial and syringe). Liraglutide QD is delivered once daily via a multiple-use pen, requires careful preparation of the medication, requires titration of the medication, and uses a thinner, shorter needle.

The attributes and levels were combined into choice sets using a published orthogonal array [25]. An orthogonal fractional factorial design was used to identify the minimum specification of the DCE experimental design in order to fairly represent combinations of the attributes and levels. These combinations were paired using a fold-over design. Each choice question 


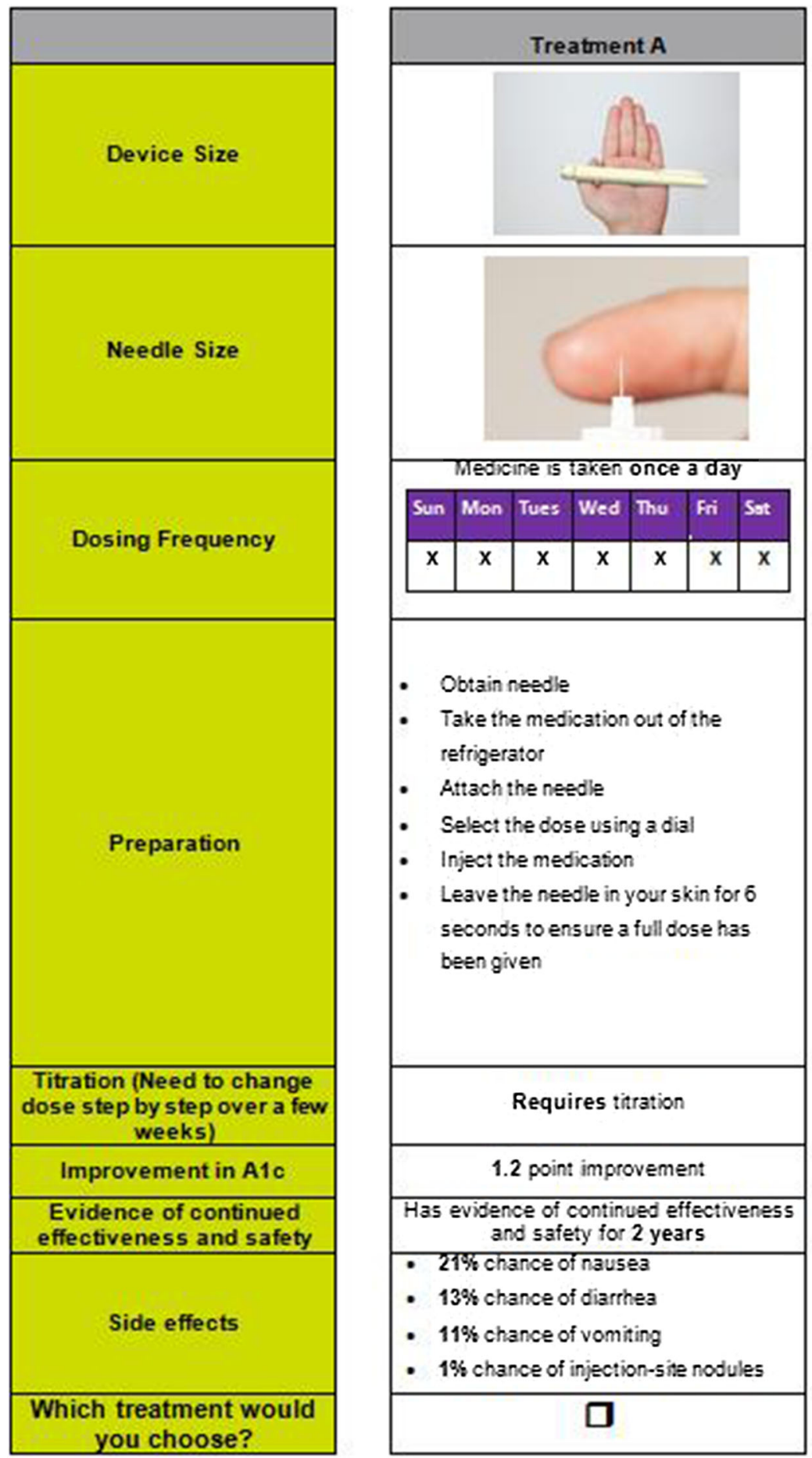

\begin{tabular}{|l|}
\hline \multicolumn{2}{|c|}{ Treatment $B$} \\
\hline \\
\hline
\end{tabular}

Fig. 1 Example of a discrete choice experiment question comparing treatment profiles reflecting liraglutide QD and exenatide QW (Treatment A vs. Treatment B)

presented two hypothetical treatments (Treatment $\mathrm{A}$ or B) and participants were asked to indicate their preferred choice. On the basis of the orthogonal fractional factorial design, a total of 32 pairs of choice sets were generated, with each respondent being presented with a set 
of 16 unique paired choice sets [20]. An example of a choice set included in the survey is provided in Fig. 1.

The first section of the survey consisted of questions on past and current medication use, adherence, clinical history, and demographic information. The adherence questions asked about frequency of missing, skipping, or being late in taking their oral medications and what aspect of their current medications made it most difficult or inconvenient to take as prescribed. In addition, participants were asked hypothetical questions about the potential impact of dosing frequency on their adherence.

The second section of the survey included the DCE questions. The DCE began by presenting participants with descriptions of the attributes and the levels included in the survey, followed by the 16 paired comparison questions. The English-language survey was pilot-tested with five T2DM patients in the UK, and revisions were made to improve clarity and assess cognitive and overall burden. The survey was then translated into Portuguese (Brazil), Chinese (China), German (Germany), and Japanese (Japan). Two native speakers in each country reviewed the translations for accuracy and clarity.

\section{Data Analysis}

Descriptive statistics using SAS (v.4.3) were performed to calculate means, standard deviations, ranges, frequencies, and percentages as appropriate, for the demographic and clinical characteristics data, as well as self-reported data on adherence to medication. SAS (v.4.3) was also used to analyze the DCE data using a conditional or mixed logit model, with each attribute and level included as a separate variable in the model. The choice of treatment was the dependent variable and was analyzed conditionally for each choice set (i.e., each treatment choice the respondent had to make). The conditional logistic model provided regression coefficients (i.e., utility estimates), which were then presented as odds ratios (ORs) and their 95\% confidence intervals (CI) in order to indicate the importance of a given attribute level versus a reference attribute level, as well as the likelihood of a respondent choosing a treatment with a given level of an attribute rather than a treatment with the reference level of the attribute. All analyses were performed for the total sample and separately for each country.

Sensitivity analyses were conducted to examine how much a product's overall preference was improved or worsened by changing certain attributes, while holding all other attributes at constant base case levels. For example, the exenatide QW profile was compared to the liraglutide QD profile assuming better efficacy for liraglutide QD versus exenatide QW. The exenatide QW profile assuming an auto-injector device size and auto-injector preparation was also compared to the liraglutide QD profile.

\section{RESULTS}

\section{Sample Characteristics}

A total of 1482 injection-naïve respondents completed the survey (Table 1). The mean age of patients in the study was 56 years (range $18-87$ years) and $68 \%$ were male. The majority (52\%) was White/Caucasian, employed (55\%), and educated, with $52 \%$ having at least a college degree. The mean time since the diagnosis of T2DM was 7.0 years. The majority of participants had taken metformin to treat their diabetes (54\% current users; 38\% past users). The most commonly reported comorbidities were identified as "other" (19.7\%), cardiovascular disease $(11.8 \%)$, and mental health conditions (6.8\%); however, more than half of respondents (53.5\%) reported no other health conditions. A greater proportion of the respondents in the UK and Germany were retired. With the exception of country-related differences, the sociodemographic and clinical characteristics were similar across the countries (Table 1).

\section{Current Treatments}

Overall, most patients were taking oral medication once $(32.2 \%)$, twice $(48.8 \%)$, or three times a day (16.7\%). The majority of patients 
Table 1 Sociodemographic and clinical characteristics of injection-naïve type 2 diabetes mellitus patients

\begin{tabular}{|c|c|c|c|c|c|c|}
\hline Patient characteristics & $\begin{array}{l}\text { Total } \\
(N=1482)\end{array}$ & $\begin{array}{l}\text { Brazil } \\
(n=296)\end{array}$ & $\begin{array}{l}\text { China } \\
(n=297)\end{array}$ & $\begin{array}{l}\text { Germany } \\
(n=296)\end{array}$ & $\begin{array}{l}\text { Japan } \\
(n=296)\end{array}$ & $\begin{array}{l}\text { UK } \\
(n=297)\end{array}$ \\
\hline Mean age, years $(\mathrm{SD})$ & $56.0(11.4)$ & $51.7(12.2)$ & $49.9(9.2)$ & $58.8(9.8)$ & $57.2(11.4)$ & $62.3(9.5)$ \\
\hline Male/female (\%) & $68 / 32$ & $59 / 41$ & $54 / 46$ & $70 / 30$ & $88 / 12$ & $67 / 33$ \\
\hline \multicolumn{7}{|l|}{ Education (\%) } \\
\hline No formal qualifications & 4.6 & 1.4 & 0.0 & 9.5 & 0.3 & 11.8 \\
\hline GCSE/O-level or equivalent & 22.3 & 22.6 & 1.7 & 53.7 & 1.4 & 32.0 \\
\hline A-level or equivalent & 21.5 & 18.6 & 14.1 & 15.2 & 36.1 & 23.6 \\
\hline University degree & 43.7 & 39.2 & 81.5 & 18.2 & 56.1 & 23.2 \\
\hline Postgraduate degree & 8.0 & 18.2 & 2.7 & 3.4 & 6.1 & 9.4 \\
\hline \multicolumn{7}{|l|}{ Ethnic group (\%) } \\
\hline White & 51.6 & 61.1 & 1.3 & 97.6 & 0.0 & 97.0 \\
\hline Mixed/multiple ethnic groups & 2.8 & 12.2 & 0.7 & 1.0 & 0 & 0.0 \\
\hline Asian & 40.7 & 1.7 & 99.7 & 0.3 & 100.0 & 1.7 \\
\hline Black/African/Caribbean & 5.3 & 25.7 & 0.3 & 0.0 & 0.0 & 0.3 \\
\hline Arab & 0.2 & 0.3 & 0.3 & 0.3 & 0.0 & 0.0 \\
\hline Other & 0.2 & 0.7 & 0.0 & 0.0 & 0.0 & 0.3 \\
\hline Prefer not to answer & 0.5 & 0.3 & 0.0 & 1.0 & 0.0 & 1.0 \\
\hline \multicolumn{7}{|l|}{ Employment (\%) } \\
\hline Employed, full-time & 39.8 & 39.2 & 80.8 & 22.3 & 40.5 & 16.2 \\
\hline Employed, part-time & 7.0 & 7.8 & 1.7 & 9.8 & 10.5 & 5.4 \\
\hline Self-employed & 8.3 & 16.9 & 4.0 & 5.1 & 11.1 & 4.4 \\
\hline Student & 0.5 & 1.4 & 0.0 & 0.3 & 0.7 & 0.0 \\
\hline $\begin{array}{l}\text { Stay-at-home parent/ } \\
\text { homemaker }\end{array}$ & 2.5 & 1.0 & 0.0 & 3.4 & 4.4 & 3.7 \\
\hline Unemployed & 6.5 & 9.1 & 0.0 & 9.1 & 8.4 & 6.1 \\
\hline Retired & 31.7 & 21.6 & 13.5 & 43.6 & 22.0 & 57.9 \\
\hline Disabled & 2.8 & 0.3 & 0.0 & 6.4 & 1.7 & 5.4 \\
\hline Other & 0.9 & 2.7 & 0.0 & 0.0 & 0.7 & 1.0 \\
\hline \multicolumn{7}{|l|}{ Comorbidities (\%) } \\
\hline Cancer & 2.2 & 2.0 & 0.3 & 2.7 & 2.0 & 4.0 \\
\hline Cardiovascular condition & 11.8 & 12.8 & 3.0 & 21.3 & 9.8 & 12.1 \\
\hline Chronic kidney disease & 1.6 & 1.4 & 0.0 & 3.7 & 0.7 & 2.4 \\
\hline Mental health condition & 6.8 & 1.7 & 4.7 & 14.2 & 8.1 & 5.4 \\
\hline
\end{tabular}


Table 1 continued

\begin{tabular}{|c|c|c|c|c|c|c|}
\hline Patient characteristics & $\begin{array}{l}\text { Total } \\
(N=1482)\end{array}$ & $\begin{array}{l}\text { Brazil } \\
(n=296)\end{array}$ & $\begin{array}{l}\text { China } \\
(n=297)\end{array}$ & $\begin{array}{l}\text { Germany } \\
(n=296)\end{array}$ & $\begin{array}{l}\text { Japan } \\
(n=296)\end{array}$ & $\begin{array}{l}\text { UK } \\
(n=297)\end{array}$ \\
\hline Respiratory disorder & 5.1 & 5.1 & 0.7 & 9.1 & 3.7 & 7.1 \\
\hline Diabetic retinopathy & 3.0 & 2.0 & 4.4 & 0.3 & 2.4 & 5.7 \\
\hline Diabetic foot problems & 5.5 & 5.4 & 3.4 & 10.5 & 3.0 & 5.1 \\
\hline Diabetic neuropathy & 4.7 & 5.7 & 1.0 & 7.4 & 2.7 & 6.7 \\
\hline Other & 19.7 & 18.9 & 1.3 & 25.7 & 29.4 & 23.2 \\
\hline None & 53.5 & 54.1 & 83.2 & 35.1 & 49.3 & 45.8 \\
\hline $\begin{array}{l}\text { Time since diagnosis of T2DM, } \\
\text { years [range] }\end{array}$ & $\begin{array}{c}7.0[0.5 \\
61.9]\end{array}$ & $\begin{array}{c}6.7[0.9 \\
39.3]\end{array}$ & $\begin{array}{c}3.9[0.9 \\
49.8]\end{array}$ & $7.7[0.9,61.9]$ & $\begin{array}{c}8.5[0.9 \\
52.8]\end{array}$ & $\begin{array}{c}8.3[0.5 \\
47.1]\end{array}$ \\
\hline Mean HbAlc ${ }^{a}, \%(S D)$ & $7.4(2.3)$ & $8.3(4.0)$ & $9.1(3.5)$ & $6.7(1.1)$ & $7.0(0.9)$ & $7.5(2.7)$ \\
\hline
\end{tabular}

HbAlc glycated hemoglobin, SD standard deviation, T2DM type 2 diabetes mellitus

a $n=682$ (total), 73 (Brazil), 82 (China), 165 (Germany), 273 (Japan), and 89 (UK)

reported having no difficulty $(82.7 \%)$ or little difficulty taking oral medication (10.7\%). Overall, $46.8 \%$ of respondents indicated that they never missed a dose of their current medication. Among the remaining respondents, $8.6 \%$ missed a dose about once a year, $10.9 \%$ missed a dose about once every 6 months, $6.7 \%$ missed a dose once every 3 months, and $27.1 \%$ missed a dose at least once a month. Reasons for completely missing a dose included forgetting $(65.7 \%)$, not having the medication with them at the scheduled time (27.8\%), a change in daily routine $(13.5 \%)$, and running out of medication $(8.3 \%)$. More than half $(52.7 \%)$ reported taking medication late, with the reasons including forgetting (55.6\%), not having the medication with them at the scheduled time $(28.6 \%)$, or experiencing a change in daily routine $(21.1 \%)$.

\section{Attributes Influencing Choice of Injectable Medication}

Total sample and country-specific analyses revealed that among the eight attributes included in the survey, side effects, efficacy, and dosing frequency were the top three attributes driving patient preference. Needle size, device size, and required injection preparation were the least important predictors of choice.
Across all countries, patients indicated a preference for a treatment associated with fewer side effects (OR 2.14; 95\% CI 2.08, 2.20; $p<0.001)$, a treatment offering a 1.5-point improvement in HbA1c (OR 1.85; 95\% CI 1.77, 1.93; $p<0.001)$, and a treatment that was administered once weekly (OR 1.63; 95\% CI $1.59,1.68 ; p<0.001)$. The relative importance of these attributes varied by country. Having fewer side effects was the most highly preferred attribute in the UK (OR 3.54; $p<0.001$ ), Japan (OR 2.55; $p<0.001$ ), Germany (OR 2.39; $p<0.001$ ), and Brazil (OR 2.00; $p<0.001$ ), but not in China where a treatment offering a 1.5-point improvement in HbA1c was the most highly preferred attribute (OR 2.67; $p<0.001$ ). Efficacy and dosing frequency were ranked second and third in all countries except China where side effects was second and Japan where dosing frequency was second and efficacy was third (Table 2).

Among the five device-related attributes, dosing frequency, titration, and the preparation required were significant predictors of choice in the total sample. Patients significantly preferred weekly to daily dosing in both the total sample (see above) and in each of the individual countries, including Japan (OR 2.24; $p<0.001$ ), Brazil (OR 1.75; $p<0.001$ ), Germany (OR 1.71; 


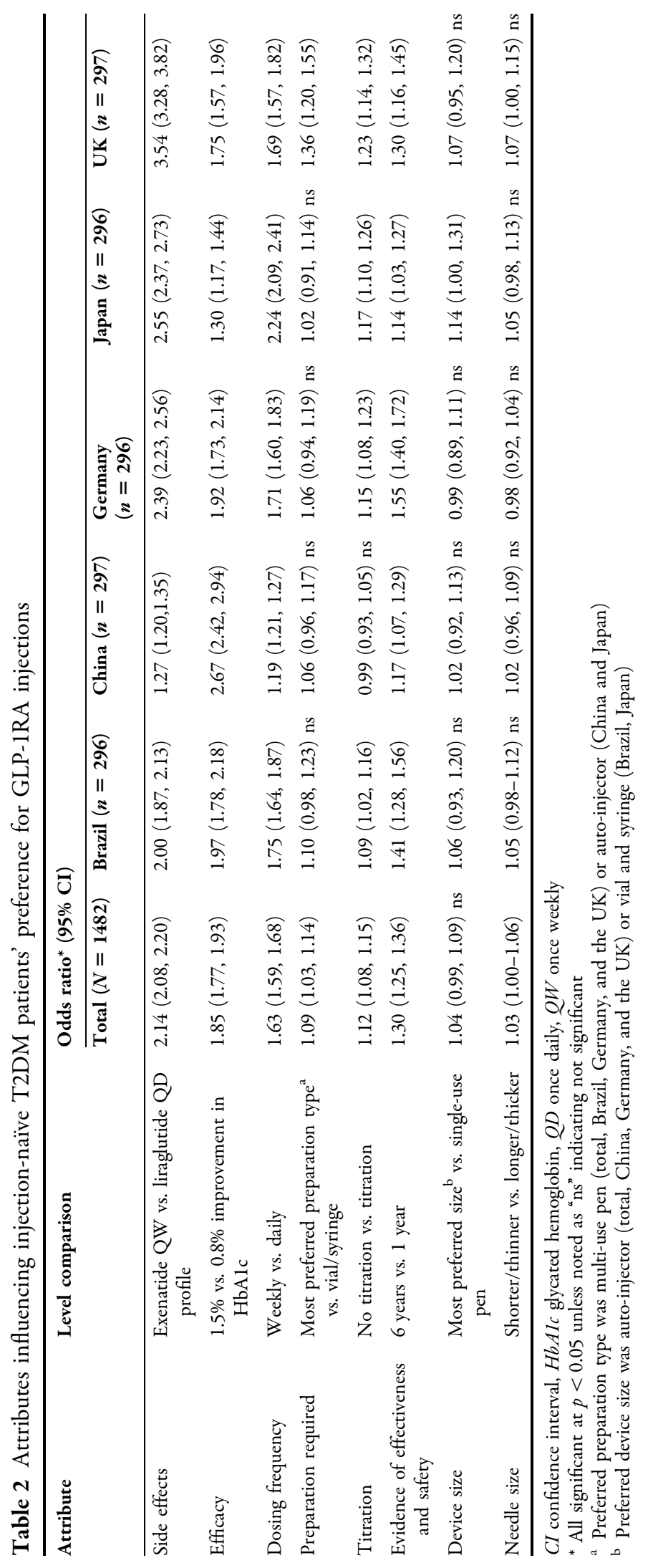


$p<0.001)$, the UK (OR 1.69; $p<0.001)$, and China (OR 1.19; $p<0.001$ ). Patients significantly preferred no titration over titration in the total sample (OR 1.12; $p<0.001$ ) and in the UK (OR 1.23; $p<0.001$ ), Japan (OR 1.17; $p<0.001$ ), Germany (OR 1.15, $p<0.001$ ), and Brazil (OR 1.09; $p=0.01$ ), but not in China (OR $0.99 ; p=0.75)$. Patients preferred the preparation associated with a multi-use pen over a vial/ syringe in the total sample (OR 1.09; $p=0.002$ ) and in the UK sample (OR 1.36; $p<0.001$ ). Needle size and device size were the least important device- and regimen-related attributes in driving preference for the total and country-specific samples.

When patients were asked about hypothetical injectable T2DM treatments, $64.2 \%$ of respondents stated that a once-weekly dose would be easiest to follow, while $31.1 \%$ selected once daily, and $4.7 \%$ thought twice daily would be easiest to follow. When injection-naïve patients were asked about the hypothetical frequency of missing a dose of an injectable diabetes treatment, $60.7 \%$ stated that they would never miss a once-weekly dose compared with $54.3 \%$ who stated they would never miss a daily dose. If the GLP-1RA injection was administered once weekly, $12.5 \%$ of respondents thought they might hypothetically miss a dose at least once every month. If the GLP-1RA injection was once daily, $22.3 \%$ of patients thought they might hypothetically miss a dose at least once every month.

\section{Comparison of Hypothetical GLP-1RA Profiles}

In order to determine the influence of attributes other than efficacy, hypothetical GLP-1 RA profiles with equal efficacy were constructed in sensitivity analyses. Total sample data showed that when efficacy was assumed to be equal (1.2-point improvement in HbA1c), injection-naïve patients favored a GLP-1RA profile approximating exenatide QW administered via a single-use pen versus a profile approximating liraglutide QD (OR 3.36; $p<0.001)$, with 77.0\% of the sample preferring the profile approximating exenatide QW. When examined by country, preference for exenatide QW was highest among the UK sample (OR 4.88; $p<0.001)$ and lowest among the Chinese sample (OR 1.60; $p<0.001$ ) (Fig. 2).

When efficacy was assumed to be better for liraglutide QD than for exenatide QW administered via a single-use pen (1.2-point improvement in HbA1c vs. 0.8-point improvement in HbA1C), total sample results showed that $70.4 \%$ of respondents preferred the profile approximating exenatide QW (OR 2.38, $p<0.001$ ). When examined by country, preference for exenatide QW single-use pen was highest among Japanese respondents (OR 4.22, $p<0.001)$. The Chinese sample showed no significant preference for one profile over the other (OR 0.90; $p=0.38$ ).

When changing exenatide QW's device type to an auto-injector rather than a single-use pen (assuming the same efficacy of a 1.2-point improvement in $\mathrm{HbA1c}$ ), the strength of preference for the profile approximating exenatide $\mathrm{QW}$ increased in the total sample (OR 4.27; $p<0.001$ ), with $81.0 \%$ of the sample preferring the profile approximating exenatide QW (auto-injector). When examining country-specific analyses, the OR indicating preference for the profile approximating exenatide QW (auto-injector) compared with the profile approximating liraglutide QD was again highest among the UK sample (OR 8.48; $p<0.001$ ) and lowest among the Chinese sample (OR 1.66; $p<0.001$ ) (Fig. 3). When efficacy was assumed to

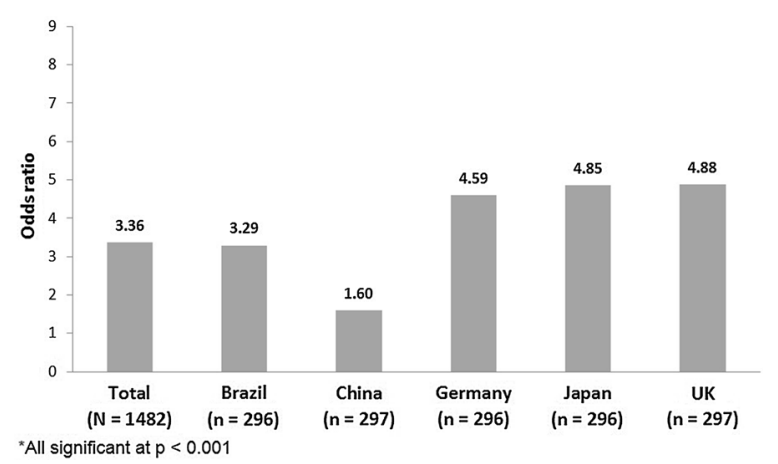

Fig. 2 Odds ratios for preferring profile approximating exenatide QW (single-use pen) versus profile approximating liraglutide QD (with equal efficacy of 1.2-point improvement in $\mathrm{HbAlc}$ ), overall and by country. $\mathrm{HbAlc}$ glycated hemoglobin, $Q D$ once daily, $Q W$ once weekly 


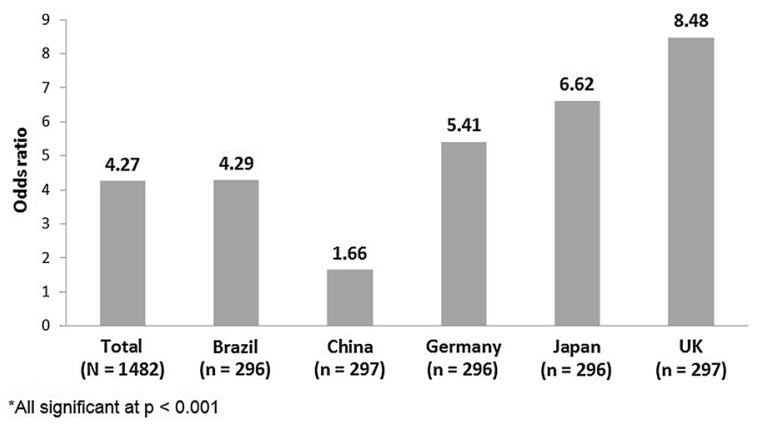

Fig. 3 Odds ratios for preferring profile approximating exenatide QW (auto-injector) versus profile approximating liraglutide QD profile (with equal efficacy of 1.2-improvement in HbAlc), overall and by country. $H b A 1 c$ glycated hemoglobin, $Q D$ once daily, $Q W$ once weekly

be better for liraglutide QD than exenatide QW administered via an auto-injector (1.2-point improvement in HbA1c vs. 0.8-point improvement in HbA1c), total sample results continued to show that there was a higher preference for the profile approximating exenatide QW in the total sample (OR 3.03, $p<0.001$ ). Similar trends were observed in the UK (OR 6.14; $p<0.001$ ), Japan (OR 5.75; $p<0.001$ ), Germany (OR 3.57; $p<0.001$ ), and Brazil (OR 3.07; $p<0.001$ ), but not in China (OR 0.94; $p=0.59$ ).

\section{DISCUSSION}

This multi-country DCE study found that side effects, efficacy, and dosing frequency were most important in influencing preferences among injection-naïve patients. Patients indicated a perception that a once-weekly dosing regimen would result in fewer missed doses than a once-daily dosing regimen. When full medication profiles exhibiting the attributes of two GLP-1RAs with differing key features were compared and efficacy was assumed to be the same, patients preferred the profile approximating exenatide QW (administered via a single-use pen or an auto-injector) over the profile approximating liraglutide QD.

Injection-naïve patients represent an important group of T2DM patients as their preferences have not been influenced by previous experiences of injecting diabetic medication [26]. Other DCE studies have also examined injection-naïve patients' preferences for the attributes of GLP-1RAs [26-28]. One of these studies also included injection-experienced patients, and focused specifically on the dosing and device-related attributes of hypothetical profiles approximating exenatide QW administered by vial/syringe or a single-use pen compared with a profile approximating liraglutide QD administered via a multi-use pen [26]. These other studies provide an important context for assessing the influence of injected GLP-1RA attributes on the preferences of patients. In these previous studies, as in the current study, patients' preferences relating to GLP1-RA medication were influenced not only by tolerability but also other dosing and device-related attributes [26-28]. Across all studies, dosing frequency was an important driver of injection-naïve patients' choice of GLP-1RA medication, with once-weekly injections prioritized over once-daily injections [26-28].

Efficacy was found to be less influential in other GLP-1RA DCE studies [27, 28] versus the current study. However, efficacy was defined differently in these other studies (i.e., achieving HbA1c <7\%) [27, 28], and the difference between the levels was very small (e.g., $67.9 \%$ vs. $68.3 \%$ [27] and $69.1 \%$ vs. $71.4 \%$ [28] of patients achieving HbA1c goal of $<7 \%$ ), thereby reducing its importance as an influential attribute. Even in this study, efficacy played a less prominent role overall when examining preferences for two different full profiles. When efficacy was assumed to be equal, patients indicated a strong preference for a GLP-1RA profile approximating exenatide QW (delivered either via a single-use pen or auto-injector) over a profile approximating liraglutide QD. Even when the profile approximating liraglutide QD was assumed to have better efficacy (1.2 vs. 0.8-point improvement in HbA1c, respectively), patients still favored the once-weekly injection profile. This preference was observed in the total sample as well as within samples from four individual countries, but not in the Chinese sample, where efficacy played a more influential role in driving preferences. The greater influence of efficacy among the Chinese sample could be related to the fact that they had the poorest glucose control compared to the other 
countries, reporting the highest mean $\mathrm{HbA} 1 \mathrm{c}$ level of $9.1 \%$. This finding of suboptimal glucose control among the Chinese sample is consistent with other studies [29-31] that have found inadequate blood glucose control among T2DM patients in China.

The various attributes of medication (including efficacy, adverse events, frequency of dosing, type of delivery device) have an important effect on T2DM patients' adherence to medication [10, 32, 33]. Currently, nonadherence to therapy is a common problem for patients with T2DM, and it has been associated with increases in morbidity, mortality, and healthcare costs $[10,11,13]$. If an appreciation and understanding of patients' preference for treatment can be gained and a patient-centered approach is used during clinical decision-making, then adherence is likely to improve [7]. If adherence can be increased, then improvements in diabetes-related outcomes will be likely, including improvements in glycemic control, and reductions in short-term and long-term diabetes-related complications, and healthcare resource utilization [10, 12, 14, 34-37]. Medication regimens with less frequent dosing regimen are associated with greater adherence to medication [38]. GLP-1RAs are no exception, with once-weekly injections of a GLP-1RA being associated with greater patient adherence than once-daily injections of a GLP-1RA [39-41].

Understanding patient preference for treatment attributes is needed not only during the clinical decision-making process [7] to improve adherence and clinical outcomes but also during the development of any diabetes medication. The US Food and Drug Administration encourages the inclusion of patient preference information during premarket approval applications and device labelling [42]. The development of GLP-1RAs has followed such a patient-centered approach. Aside from the important considerations of efficacy and side effects, consideration during the development process has been given to an attribute which this study indicated was very important to patients, namely dosing frequency. The injectable GLP-1RAs were first available on the market as twice-daily injections (e.g., exenatide), but in line with patient preferences, GLP-1RAs became available as once-daily injections (e.g., liraglutide, lixisenatide), and then as once-weekly formulations (e.g., exenatide, albiglutide, and dulaglutide). Other attributes of injectable medications (such as preparation or titration), identified in this study as being important, have been the focus of the patient-centered GLP-1RA development program $[17,43,44]$.

This study had a number of limitations. DCEs, while a common methodology in eliciting preferences, may not accurately reflect real-world decisions. Since most of the patients were recruited from market research panels, the participants from the five target countries may not be truly representative of T2DM patients in each country. This is particularly the case for the Chinese sample, where $84 \%$ of the sample had obtained a university degree or higher, which may have biased the results. Another limitation of this study is that this particular sample included attributes from specific GLP-1RA treatments, exenatide QW and liraglutide QD, thus limiting the generalizability of the findings to other GLP-1RA treatments. Liraglutide QD and exenatide QW were selected for the comparison because they reflect key differences in GLP1-RA treatments, with differences in regimen and side effects, and were available at the time of the study. Furthermore, liraglutide QD is the leading daily treatment for T2DM, and exenatide QW is the first once-weekly T2DM treatment. Nevertheless, other published studies have taken a similar approach comparing two specific treatments and have shown that results from general designs are often different than results for more specific designs involving comparisons of specific treatments [27, 28]. Additionally, subjects were asked to consider the overall tolerability profile of the treatments based on the most commonly occurring side effects, though the influence of specific side effects on preferences may have varied. Lastly, the number of attributes included in the survey was limited to attributes important to patients related to efficacy, safety, tolerability, and convenience in order to reduce cognitive burden on participants. However, there may be other aspects of treatment that could influence preference for treatment, such as out of pocket cost or time required for training on the use of the device. Thus, the results may not reflect actual market share for these 
treatments, as other factors may also influence treatment decisions.

\section{CONCLUSION}

Understanding patient preferences is important for informing new generations of T2DM treatments and ensuring patient-centered care. Since current GLP-1RA treatments vary greatly in efficacy, dosing regimen, and risk of side effects, it is important for healthcare providers to incorporate patient preferences in making treatment decisions as it may increase medication adherence and thus also improve shortand long-term clinical outcomes. The findings from this global study suggest that healthcare providers may need to take into account side effects, efficacy, and dosing frequency when prescribing medications, and drug research and development should focus on these attributes for developing new generations of treatment.

\section{ACKNOWLEDGEMENTS}

Sponsorship for this study and article processing charges were funded by AstraZeneca. All named authors meet the International Committee of Medical Journal Editors (ICMJE) criteria for authorship for this manuscript, take responsibility for the integrity of the work as a whole, and have given final approval to the version to be published. Editorial assistance in the preparation of this manuscript was provided by Monique Curran. Support for this assistance was funded by AstraZeneca. The preliminary results from this study were previously presented at the European Association for the Study of Diabetes (EASD) Annual Meeting 2016 and the 2016 American Diabetes Association (ADA) 76th Scientific Sessions.

Disclosures. Lei Qin is employed by AstraZeneca.

Stephanie Chen is employed by AstraZeneca. Susan Grandy is employed by AstraZeneca. Alka Shaunik was employed by AstraZeneca at the time of this study.
Emuella Flood is employed by ICON plc, which was commissioned by AstraZeneca to perform the study.

Beverly Romero is employed by ICON plc, which was commissioned by AstraZeneca to perform the study.

Marie de la Cruz is employed by ICON plc, which was commissioned by AstraZeneca to perform the study.

Cynthia Alvarez is employed by ICON plc, which was commissioned by AstraZeneca to perform the study.

Compliance with Ethics Guidelines. The study protocol was approved by the Salus International Review Board (Austin, TX, USA). All procedures followed were in accordance with the ethical standards of the responsible committee on human experimentation (institutional and national) and with the Helsinki Declaration of 1964, as revised in 2013. Informed consent was obtained from all patients for being included in the study.

Data Availability. The datasets during and/ or analyzed during the current study are available from the corresponding author on reasonable request.

Open Access. This article is distributed under the terms of the Creative Commons Attribution-NonCommercial 4.0 International License (http://creativecommons.org/licenses/ by-nc/4.0/), which permits any noncommercial use, distribution, and reproduction in any medium, provided you give appropriate credit to the original author(s) and the source, provide a link to the Creative Commons license, and indicate if changes were made.

\section{REFERENCES}

1. Fonseca VA. Defining and characterizing the progression of type 2 diabetes. Diabetes Care. 2009;32(Suppl 2):S151-6.

2. American Diabetes Association. Classification and diagnosis of diabetes. Diabetes Care. 2016;39(Suppl 1):S13-22. 
3. International Diabetes Federation. IDF diabetes atlas, 7th ed. 2015. http://www.diabetesatlas.org. Accessed 1 Sept 2016.

4. Shaw JE, Sicree RA, Zimmet PZ. Global estimates of the prevalence of diabetes for 2010 and 2030. Diabetes Res Clin Pract. 2010;87:4-14.

5. Jaacks LM, Siegel KR, Gujral UP, Narayan KM. Type 2 diabetes: a 21st century epidemic. Best Pract Res Clin Endocrinol Metab. 2016;30:331-43.

6. Ryden L, Grant PJ, Anker SD, et al. ESC guidelines on diabetes, pre-diabetes, and cardiovascular diseases developed in collaboration with the EASDsummary. Diabetes Vasc Dis Res. 2014;11:133-73.

7. Inzucchi SE, Bergenstal RM, Buse JB, et al. Management of hyperglycemia in type 2 diabetes, 2015: a patient-centered approach. Update to a position statement of the American Diabetes Association and the European Association for the Study of Diabetes. Diabetes Care. 2015;38:140-9.

8. Chamberlain JJ, Rhinehart AS, Shaefer CF Jr, Neuman A. Diagnosis and management of diabetes: synopsis of the 2016 American Diabetes Association Standards of Medical Care in Diabetes. Ann Intern Med. 2016;164:542-52.

9. American Diabetes Association. 7. Approaches to glycemic treatment. Diabetes Care. 2016;39(Suppl 1):S52-9.

10. Garcia-Perez LE, Alvarez M, Dilla T, Gil-Guillen V, Orozco-Beltran D. Adherence to therapies in patients with type 2 diabetes. Diabetes Ther. 2013;4:175-94.

11. Blackburn DF, Swidrovich J, Lemstra M. Non-adherence in type 2 diabetes: practical considerations for interpreting the literature. Patient Prefer Adher. 2013;7:183-9.

12. Simpson SH, Lin M, Eurich DT. Medication adherence affects risk of new diabetes complications: a cohort study. Ann Pharmacother. 2016;50:741-6.

13. Curkendall SM, Thomas N, Bell KF, Juneau PL, Weiss AJ. Predictors of medication adherence in patients with type 2 diabetes mellitus. Curr Med Res Opin. 2013;29:1275-86.

14. Asche C, LaFleur J, Conner C. A review of diabetes treatment adherence and the association with clinical and economic outcomes. Clin Ther. 2011;33:74-109.

15. Garber AJ. Novel GLP-1 receptor agonists for diabetes. Expert Opin Investig Drugs. 2012;21:45-57.
16. Ostergaard L, Frandsen CS, Madsbad S. Treatment potential of the GLP-1 receptor agonists in type 2 diabetes mellitus: a review. Expert Rev Clin Pharmacol. 2016;9:241-65.

17. Madsbad S. Review of head-to-head comparisons of glucagon-like peptide-1 receptor agonists. Diabetes Obes Metab. 2016;18:317-32.

18. Triplitt C, Solis-Herrera C. GLP-1 receptor agonists: practical considerations for clinical practice. Diabetes Educ. 2015;41:32s-46s.

19. Trujillo JM, Nuffer W, Ellis SL. GLP-1 receptor agonists: a review of head-to-head clinical studies. Ther Adv Endocrinol Metab. 2015;6:19-28.

20. Ryan M, Bate A, Eastmond CJ, Ludbrook A. Use of discrete choice experiments to elicit preferences. Qual Health Care. 2001;10(Suppl 1):i55-60.

21. de Bekker-Grob EW, Ryan M, Gerard K. Discrete choice experiments in health economics: a review of the literature. Health Econ. 2012;21:145-72.

22. Grandy S, Chen S, Flood E, Romero B, Bergenheim K, Ryden A. GLP-1 receptor agonist device- and regimen-related features important to injection-experienced and injection-naïve patients with T2DM: a multi-national interview study. In: ePoster \#905. 51st European Association for the Study of Diabetes annual meeting, 15-18 Sept 2015, Stockholm.

23. Novo Nordisk A/S. Liraglutide once daily (Victoza): package insert. Denmark: Bagsvaerd; 2010.

24. Amylin Pharmaceuticals Inc. Exenatide once weekly (Bydureon): package insert. San Diego: Amylin Pharmaceuticals; 2012.

25. Sloane NJA. A library of orthogonal arrays. 2016. http://neilsloane.com/oadir/. Accessed 18 Aug 2016.

26. Hauber AB, Nguyen H, Posner J, Kalsekar I, Ruggles J. A discrete-choice experiment to quantify patient preferences for frequency of glucagon-like peptide-1 receptor agonist injections in the treatment of type 2 diabetes. Curr Med Res Opin. 2016;32:251-62.

27. Gelhorn HL, Poon JL, Davies EW, Paczkowski R, Curtis SE, Boye KS. Evaluating preferences for profiles of GLP-1 receptor agonists among injection-naive type 2 diabetes patients in the UK. Patient Prefer Adher. 2015;9:1611-22.

28. Gelhorn HL, Bacci ED, Poon JL, Boye KS, Suzuki S, Babineaux SM. Evaluating preferences for profiles of glucagon-like peptide-1 receptor agonists among injection-naive type 2 diabetes patients in Japan. Patient Prefer Adher. 2016;10:1337-48. 
29. Xu Y, Wang L, He J, et al. Prevalence and control of diabetes in Chinese adults. JAMA. 2013;310:948-59.

30. Ji LN, Lu JM, Guo XH, et al. Glycemic control among patients in China with type 2 diabetes mellitus receiving oral drugs or injectables. BMC Public Health. 2013;13:602.

31. Zhang SL, Chen ZC, Yan L, Chen LH, Cheng H, Ji LN. Determinants for inadequate glycaemic control in Chinese patients with mild-to-moderate type 2 diabetes on oral antidiabetic drugs alone. Chin Med J (Engl). 2011;124:2461-8.

32. Davies MJ, Gagliardino JJ, Gray LJ, Khunti K, Mohan V, Hughes R. Real-world factors affecting adherence to insulin therapy in patients with type 1 or type 2 diabetes mellitus: a systematic review. Diabet Med. 2013;30:512-24.

33. Meece J. Improving medication adherence among patients with type 2 diabetes. J Pharm Pract. 2014;27:187-94.

34. Boye KS, Curtis SE, Lage MJ, Garcia-Perez LE. Associations between adherence and outcomes among older, type 2 diabetes patients: evidence from a Medicare supplemental database. Patient Prefer Adher. 2016;10:1573-81.

35. Buysman EK, Liu F, Hammer M, Langer J. Impact of medication adherence and persistence on clinical and economic outcomes in patients with type 2 diabetes treated with liraglutide: a retrospective cohort study. Adv Ther. 2015;32:341-55.

36. Doggrell SA, Warot S. The association between the measurement of adherence to anti-diabetes medicine and the HbA1c. Int $J$ Clin Pharm. 2014;36:488-97.

37. Holman RR, Bethel MA, George J, et al. Rationale and design of the EXenatide Study of
Cardiovascular Event Lowering (EXSCEL) trial. Am Heart J. 2016;174:103-10.

38. Richter A, Anton SF, Koch P, Dennett SL. The impact of reducing dose frequency on health outcomes. Clin Ther. 2003;25:2307-35 (discussion 6).

39. Johnston SS, Nguyen H, Felber E, et al. Retrospective study of adherence to glucagon-like peptide-1 receptor agonist therapy in patients with type 2 diabetes mellitus in the United States. Adv Ther. 2014;31:1119-33.

40. Qiao Q, Ouwens MJ, Grandy S, Johnsson K, Kostev K. Adherence to GLP-1 receptor agonist therapy administered by once-daily or once-weekly injection in patients with type 2 diabetes in Germany. Diabetes Metab Syndr Obes. 2016;9:201-5.

41. Yu M, Xie J, Fernandez Lando L, Kabul S, Swindle RW. Liraglutide versus exenatide once weekly: persistence, adherence, and early discontinuation. Clin Ther. 2016;38:149-60.

42. US Food and Drug Administration. Patient preference information-voluntary submission, review in premarket approval applications, humanitarian device exemption applications, and de novo requests, and inclusion in decision summaries and device labeling. 2016. http://www.fda.gov/downloads/medicaldevices/ deviceregulationandguidance/guidancedocuments/ ucm446680.pdf. Accessed 15 Sept 2016.

43. Mittermayer F, Caveney E, De Oliveira C, et al. Addressing unmet medical needs in type 2 diabetes: a narrative review of drugs under development. Curr Diabetes Rev. 2015;11:17-31.

44. Trujillo JM, Nuffer W. GLP-1 receptor agonists for type 2 diabetes mellitus: recent developments and emerging agents. Pharmacotherapy. 2014;34:117486. 